\title{
Artículos de Reporte de caso
}

Orientación espacial: una ruta de enseñanza y aprendizaje centrada en ubicaciones y trayectorias

Jeimmy Catalina Zapateiro Segura, Soor Katharine Poloche Arango, Leonor Camargo Uribe

Medición de puntos GPS por el método estático con equipo diferencial. Una experiencia didáctica en el Instituto Pedagógico de Maturín

Eucaris del Carmen Agüero Corzo, Argenis de Jesús Montilla Pacheco, Gerardo José Valero Segovia

Validación y aplicación de un test modificado de Vandenberg y Kuse de rotación mental para simetría molecular

Johan Manuel Sepúlveda García, Andrés Mauricio Suárez Giraldo, José Mauricio Rodas Rodríguez, Francisco Javier Ruiz Ortega, Maria Delfa Henao Henao

Astronomía para la educación: "de Macondo al cielo, del cielo a Macondo" 



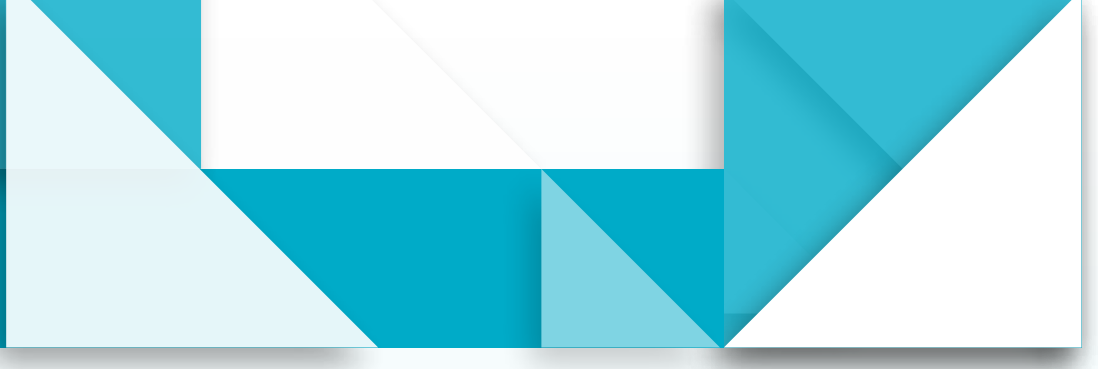

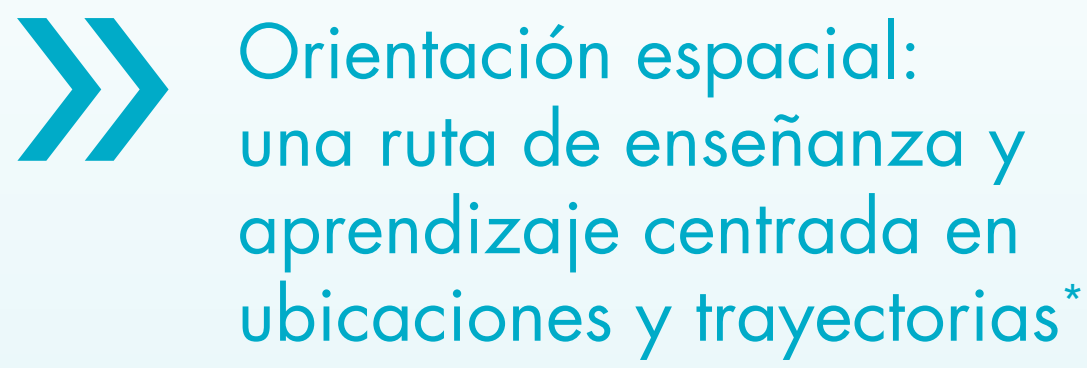

- Spatial Orientation: A Learning and Teaching Method Focused on Paths and Locations

- Orientação espacial: uma rota de ensino e aprendizagem focalizada em localizações e trajetórias

\section{Resumen}

El objetivo del reporte educativo fue la elaboración de una propuesta didáctica para favorecer el desarrollo de la orientación espacial, a través de juegos. La metodología empleada para su desarrollo incluyó un estudio teórico sobre la orientación espacial y el juego como recurso didáctico, el diseño de los juegos, la ejecución de pruebas piloto, la observación y la reflexión de la implementación. Como conclusión principal del trabajo afirmamos que los juegos diseñados son útiles para incentivar, desarrollar y fortalecer aspectos de la orientación espacial en cada nivel de la competencia. Su implementación fortalece en los estudiantes competencias necesarias para establecer sistemas de referencia. Con el conjunto de descriptores y los juegos propuestos ofrecemos una opción para el aula de matemáticas que busca favorecer el desarrollo de la competencia orientación espacial. Por otra parte, evidenciamos la importancia de usar el juego como recurso didáctico para la enseñanza y el aprendizaje de las matemáticas.

Palabras clave

pensamiento espacial; niveles de competencia; propuesta didáctica; sistemas de referencia
Jeimmy Catalina Zapateiro Segura*

Soor Katharine Poloche Arango*** Leonor Camargo Uribe

** Licenciada en Matemáticas. Universidad Pedagógica Nacional. Bogotá, Colombia.

dma_izapateiro413@pedagogica.edu.co

ORCID: http://orcid.org/0000-0003-1679-9665.

*** Licenciada en Matemáticas. Universidad Pedagógica Nacional. Bogotá, Colombia.

dma_spoloche014@pedagogica.edu.co

ORCID: http://orcid.org/0000-0002-9068-2820.

**** Doctora en Didáctica de las Matemáticas de la Universidad de Valencia, España. Profesora e investigadora del Departamento de Matemáticas de la Universidad Pedagógica Nacional. Bogotá, Colombia.

Icamargo@pedagogica.edu.co

ORCID: hHtp://orcid.org/0000-0002-2237-7306.

Una versión preliminar de este artículo ha sido publicada en las memorias del II Encuentro de Investigación en Educación Matemática, Universidad del Atlántico, Puerto Colombia, 20 y 21 de octubre de 2016.

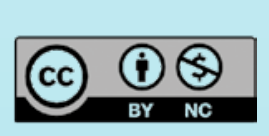

Primer semestre de 2018 / ISSN 0121 - 3814 pp. $119-136$ 


\begin{abstract}
The purpose of the educational report was to elaborate a didactic proposal to favor the development of spatial orientation through games. The methodology used to develop it included a theoretical study on spatial orientation and games as a teaching resource, the design of the games, the implementation of pilot tests, observation of and reflection on the implementation. As a main conclusion, we sustain that the designed games are useful to encourage, develop and strengthen aspects of spatial orientation in each competence level. Its implementation strengthens the competences that students require to establish reference systems. With the set of descriptors and proposed games, we offer an option for the math classroom that seeks to favor the development of the spatial orientation competence. On the other hand, we demonstrate the importance of using games as an educational resource for teaching and learning math.
\end{abstract}

Keywords

spatial thinking; competence levels; teaching proposal; reference systems

\title{
Resumo
}

objetivo do relatório educativo foi a elaboração de uma proposta didática para favorecer o desenvolvimento da orientação espacial através de jogos. A metodologia utilizada para seu desenvolvimento incluiu um estudo teórico sobre a orientação espacial e o jogo como recurso didático, o design dos jogos, a execução de provas piloto, a observação e a reflexão da implementação. Como principal conclusão do trabalho, afirmamos que os jogos construídos são úteis para incentivar, desenvolver e fortalecer aspectos da orientação espacial em cada nível da competência. Sua implementação fortalece, nos estudantes, competências necessárias para estabelecer sistemas de referência. Com o conjunto de descritores e os jogos propostos, oferecemos uma opção para a ala de matemáticas que visa favorecer o desenvolvimento da competência orientação espacial. Por outro lado, evidenciamos a importância de utilizar o jogo como recurso didático para o ensino e a aprendizagem das matemáticas.

Palavras-chave

pensamento espacial; níveis de competência; proposta didática; sistemas de referência 


\section{Introducción}

En la cotidianidad y en el ámbito educativo se presentan una variedad de situaciones problema relacionadas con ubicaciones, localizaciones y trayectorias que, muy seguramente, con la ayuda de la geometría se pueden resolver. Desde este punto de vista, tiene importancia la enseñanza y aprendizaje de esta área de las matemáticas, que abarca múltiples dimensiones. Una de ellas es la orientación espacial, la cual ha desempeñado un papel esencial desde el origen de la humanidad. Se asocia con la necesidad de los individuos de ubicarse y moverse en el espacio para poder actuar en el entorno que los rodea.

En este artículo presentamos una propuesta de enseñanza y aprendizaje para promover el desarrollo de la orientación espacial, mediante la utilización de juegos. Inicialmente hacemos una breve descripción de la situación problemática que queremos atender, la cual se centra en los bajos desempeños en orientación espacial que hay en la población colombiana. Seguidamente, exponemos aspectos principales del marco de referencia, los cuales se constituyeron en el pilar de la propuesta; allí presentamos consideraciones de carácter teórico, referidas a la orientación espacial y a los juegos como recurso didáctico. Luego, describimos de manera general la propuesta y cada una de las fases llevadas a cabo en la elaboración de la misma. Posteriormente, presentamos los juegos y algunas reflexiones sobre su ejecución, fruto de una implementación piloto.

\section{Descripción de la problemática}

Los niveles de competencia en matemáticas alcanzados por los estudiantes colombianos no son los mejores. Los resultados de las pruebas Saber (Secretaría de Educación Distrital, SED, 2014) señalan que muy pocos estudiantes de grado quinto, noveno y once alcanzan los niveles satisfactorio y avanzado de competencia, especialmente en geometría. Al revisar los resultados de la prueba PISA-2012 (Organización para la Cooperación y el Desarrollo Económicos, OCDE, 2013, 2014), verificamos que, en promedio, los estudiantes del país han ocupado los últimos puestos y que, particularmente en geometría, no obtienen buenos puntajes.

Al indagar sobre el tipo de competencias matemáticas que se miden en las evaluaciones externas, encontramos que una de estas está relacionada con el espacio y la forma, la cual incluye desempeños propios de la orientación espacial, como la elaboración y lectura de mapas, la descripción de transformaciones en el plano y la localización de objetos o figuras en el plano de acuerdo con instrucciones dadas. Los estudiantes no tuvieron buenos desempeños y se hace un llamado a los administradores de currículos y profesores para aunar esfuerzos en busca de estrategias que mejoren las competencias de niños y jóvenes.

Los bajos desempeños en orientación espacial no solamente afectan los desempeños en el área de matemáticas. También influyen negativamente en otras áreas de conocimiento y en la vida diaria. Por ejemplo, en ciencias sociales, según Rodríguez (2011), es común encontrar en clase de geografía, niños con dificultades para elaborar mapas o planos de su entorno e incluso, trazar una ruta de su casa al colegio. Igualmente, hay estudiantes que tienen dificultades para localizar, situar puntos y orientar objetos en el plano.

Probablemente, como influencia de la falta de desarrollo de la orientación espacial, es común observar a transeúntes con dificultades para ubicarse. Buscar una dirección o localizar un lugar les resulta complejo, por fallas en ubicación espacial y en el manejo de 
coordenadas. De forma similar, hay personas que no logran elegir trayectorias de forma eficiente para llegar a un lugar determinado. Por ejemplo, en el sistema capitalino de transporte colombiano, se ha identificado que hay jóvenes y adultos que no comprenden el mapa que está a su disposición; por ello solicitan habitualmente la ayuda de otras personas para que los asesoren, no solo en la búsqueda de una ruta que los pueda llevar a su lugar de destino, sino también en la elección de la vía más eficiente.

Esta problemática quizás es una consecuencia de las falencias que existen en el orden administrativo de la educación colombiana. En los Lineamientos curriculares de Matemáticas (Ministerio de Educación Nacional, MEN, 1998), Estándares básicos de competencias en Matemáticas (MEN, 2006), y Derechos básicos de aprendizaje (MEN, 2015), no se propone un tratamiento sistemático para desarrollar la orientación espacial, aunque sí se alude a la importancia de su enseñanza. Por ejemplo, en los Lineamientos curriculares de Matemáticas (MEN, 1998), se expone que:

[...] el pensamiento espacial es esencial para el pensamiento científico, ya que es usado para representar y manipular información en el aprendizaje y en la resolución de problemas. El manejo de información para resolver problemas de ubicación, orientación y distribución de espacios es peculiar a esas personas que tienen desarrollada su inteligencia espacial. Se estima que la mayoría de profesiones científicas y técnicas, tales como el dibujo técnico, la arquitectura, las ingenierías, la aviación, y muchas disciplinas científicas como química, física, matemáticas, requieren personas que tengan un alto desarrollo de inteligencia espacial. (p. 37).

Como se puede apreciar, en los lineamientos menciona la necesidad de desarrollar la orientación espacial, pero no se encuentran directrices que puedan servir como herramienta para que los docentes la estimulen en las aulas. El desarrollo de esta competencia es un objetivo que debería estar incluido en los diseños curriculares, por sus aplicaciones prácticas e implicaciones en el desarrollo cognitivo en los estudiantes. Sin embargo, la enseñanza del tema no es una tarea fácil para los profesores, según revelan diversas investigaciones (Tartre, 1990; Gonzato y Godino, 2010; Gonzato, Fernández y Díaz, 2011 ; Macías y Quintero, 2013). De acuerdo con ello, este trabajo pretende servir como herramienta de referencia para futuras investigaciones centradas en la orientación espacial, y como guía para los docentes. Específicamente, en aspectos de ubicaciones y trayectorias por ser temas poco desarrollados curricularmente. 


\section{Referentes teóricos}

\section{Consideraciones referidas a la orientación espacial}

Las maneras de pensar de los seres humanos se adquieren a través de las actividades que realizan en diferentes situaciones cotidianas; con el tiempo y la experiencia, estas actividades pueden contribuir al desarrollo de ciertas competencias con las cuales se puede llegar a tener un apropiado desempeño social y académico para convertirse en ciudadanos útiles. Una de tales formas de pensar caracterizada investigativamente es el pensamiento espacial. Según Tartre (1990) y Sarama y Clements (2009), este inicia su desarrollo desde los primeros años de la infancia y es la base de dos tipos de competencias: la orientación espacial y la visualización espacial. Dado que el trabajo se enfoca en la orientación espacial, a continuación, solo haremos referencia a este tipo de competencia.

La orientación espacial es la competencia que involucra establecer diferentes posiciones en el espacio y operar con ellas; incluye la propia posición y sus movimientos, además, las posiciones de otras personas o de objetos, representadas en mapas y coordenadas. Sarama y Clements (2009) identifican cuatro niveles de competencia que conforman el desarrollo de la orientación espacial: la ubicación espacial y la trayectoria intuitiva; la organización espacial; los modelos y mapas; y las coordenadas y la estructuración espacial.

\section{Ubicación espacial y trayectoria intuitiva}

Este nivel se refiere al desarrollo de evocaciones mentales que implican elaborar dos tipos de sistemas de referencia: el basado en claves internas y el basado en claves externas
(Newcombe y Huttenlocher, 2000; citado en Sarama y Clements, 2009). Ambos sistemas se construyen a partir del punto de vista de la posición personal. El sistema de referencia basado en claves internas está relacionado con la construcción del espacio egocéntrico del cual habla Piaget. Según Newcombe y Huttenlocher (2000; citado en Sarama y Clements, 2009), se produce al registrar en la mente una ruta o una ubicación, de acuerdo con un patrón de movimiento el cual se asocia a un objetivo a alcanzar. La ruta se codifica integrando ubicaciones con base en distancias aproximadas, direcciones y la propia posición al moverse. El sistema de referencia basado en claves externas, en este nivel, se orienta por estructuras presentes en el medio ambiente, que se usan como puntos de referencia en escenarios cercanos a la cotidianidad de las personas. Según Acredolo (1978; citado en Sarama y Clements, 2009), las personas se basan en sus experiencias exitosas para localizar objetos que se encuentran a su alrededor. Siguen rutas que se configuran a partir de puntos de referencia externos, en un proceso de interrelación entre ellos y los objetos que llaman su atención; con ello construyen evocaciones mentales del espacio.

\section{Organización espacial}

Este nivel hace referencia al desarrollo de la perspectiva espacial y las trayectorias espaciales en entornos no cercanos. El desarrollo de la perspectiva espacial consiste en la construcción de sistemas de referencia icónicos, usando puntos de referencia externos a la persona, con los cuales puede ubicarse, ubicar objetos o lugares. Los sistemas de referencia permiten una ubicación que involucra varios puntos de vista posibles; es decir, no solo se tiene en cuenta la perspectiva personal, también la de otros observadores. Según Piaget e Inhelder 
(citados en Sarama y Clements, 2009), la coordinación de diferentes puntos de vista sobre los objetos es un requisito básico para la construcción de relaciones proyectivas simples; es decir, aquellas que tienen que ver con las relaciones de posición entre objetos. Cuando se construye este tipo de perspectiva espacial es posible tener éxito en tareas como reproducir un escenario desde el punto de vista de otro observador. A diferencia de la construcción de una evocación mental, el desarrollo de las trayectorias espaciales en espacios amplios requiere ir más allá de reproducir rutas intuitivamente. Se trata de construir representaciones integradas de rutas, conectando series de puntos de referencia. Con el desarrollo de la organización espacial, según Newcombe y Huttenlocher (2000; citado en Sarama y Clements, 2009), las personas pueden planificar trayectos eficientes en situaciones que implican ubicaciones que están o no a la vista, comparando las rutas para buscar la más corta o cercana a la meta.

\section{Modelos y mapas}

Este nivel hace referencia a la creación y utilización de modelos y mapas que pueden ser útiles para localizar objetos circundantes o hacer recorridos. Para dar sentido a estos mapas, según Newcombe y Huttenlocher (2000; citado en Sarama y Clements, 2009), las personas deben crear relaciones entre los atributos geométricos y sus correspondencias con respecto a atributos físicos, ya que estos varían en escala y perspectiva. Esta relación se lleva a cabo a través de un proceso de matematización; es decir, de la realización de un modelo sencillo mediante la utilización de escalas, distancias, perspectivas y correspondencia geométrica. Según los investigadores, cuando las personas logran comprender la relación geométrica entre los mapas y el espacio real, pueden comenzar a interpretar y razonar sobre los mapas para planificar trayectorias.

\section{Coordenadas y estructuración espacial}

Este nivel hace referencia a la comprensión de las relaciones espaciales que se representan mediante la utilización de coordenadas euclidianas o polares en planos bidimensionales o tridimensionales, las cuales pueden representar ubicaciones o trayectorias de objetos en determinados puntos del plano o el espacio. Para comprender el espacio como un sistema coordenado, es necesario aprender a operar mentalmente con una organización para un objeto o conjunto de objetos en el espacio. Este proceso ha sido denominado por Sarama y Clements (2009) estructuración espacial. Según estos investigadores, se ha observado que los estudiantes primero ven una cuadrícula como una colección de cuadros en lugar de un conjunto de líneas perpendiculares. Ellos comienzan a ver gradualmente los cuadros como una organización de filas y columnas, identificando relaciones de orden y distancia dentro de la cuadrícula. Con ello se puede dar inicio a la comprensión de relaciones espaciales que se representan a través de la utilización de coordenadas, mediante etiquetas relacionadas a las 
líneas de la cuadrícula y en forma de pares ordenados de coordenadas asignados a los puntos de intersección de las líneas. Estas etiquetas deben ser integradas con el orden de la cuadrícula y con las relaciones de distancia, de modo que constituyan objetos numéricos y así poder operar con ellos, con el fin de lograr relacionar y entender las etiquetas como un sistema matemático.

\section{Consideraciones referidas a los juegos como recurso didáctico}

El juego es considerado como uno de los recursos didácticos más importantes para el aprendizaje de los niños. A través de juegos, logran su desarrollo psicomotor, cognitivo y socioafectivo. Asimismo, estimulan su creatividad y les facilita entender el mundo que les rodea (Nerea, 2013). Cabe resaltar, que los juegos no solo aportan al desarrollo de los niños. Según Blatner y Blatner (1997; citado en Muñiz, Alonso y Rodríguez, 2014), implican una serie de procesos que contribuyen al desarrollo integral, emocional y social de jóvenes y adultos.

Algunos autores han identificado las ventajas de emplear el juego en las aulas de clase. Por ejemplo, De Guzmán (1996; citado en Chamoso, Durán, García, Lalanda y Rodríguez, 2004) afirma que los juegos en la enseñanza de las matemáticas rompen con la rutina de ejercicios mecánicos y despiertan el interés de los estudiantes garantizando el esfuerzo que requiere la investigación matemática; desarrollan habilidades, destrezas, hábitos y actitudes positivas frente al trabajo escolar. Además, permiten la comprensión de procedimientos matemáticos y facilitan esquivar el rechazo de algunos estudiantes hacia las matemáticas; favorecen al desarrollo social de los estudiantes, ya que estimulan la relación con otras personas, el trabajo en equipo, la aceptación de normas, la comunicación y discusión de ideas, el reconocimiento del éxito y la comprensión de los fallos; ayudan a cambiar el rol de los estudiantes en el aula, favoreciendo una instrucción más cooperativa, donde todos los estudiantes pueden manipular, aprender y enseñar.

Para que el juego tenga un impacto positivo en la enseñanza, según Chamoso et al. (2004), el objetivo no debe ser jugar, sino utilizar los juegos como instrumentos para conseguir los objetivos que se pretenden. Además, deben incorporarse en el aula de modo meditado, planificado y con una programación previa que tenga en cuenta factores del proceso de enseñanza y aprendizaje. Por otra parte, es necesario que se implementen en el aula juegos que obliguen a pensar y reflexionar ante diversas posibilidades de actuación, investigar la mejor manera de actuar, establecer conjeturas y justificarlas para intentar convencer a los demás. Dicho en otras palabras, son preferibles los juegos en los que prevalece el desarrollo de capacidades mentales, ya sean deductivas, inductivas, experimentales, de síntesis, análisis, entre otros (Chamoso et al., 2004).

\section{Propuesta para promover el desarrollo de la orientación espacial}

La propuesta de enseñanza y aprendizaje consta de un conjunto de descriptores y cuatro juegos asociados a los niveles de competencia expuestos por Sarama y Clements (2009). Estos pueden ser una herramienta para los docentes como parte del núcleo temático del pensamiento espacial y sistemas geométricos. 


\section{Descriptores para cada nivel de la competencia}

Con base en los cuatro niveles de competencia sugeridos por Sarama y Clements (2009), elaboramos un conjunto de descriptores (tabla 1), con el fin de caracterizar el aprendizaje de la orientación espacial. A medida que se avanza de un nivel a otro se presenta una mayor exigencia cognitiva, que implica las competencias de los niveles anteriores. La propuesta de descriptores es una alternativa a la manera de cómo están construidos los aspectos de orientación espacial en los currículos colombianos, ya que en ellos no se ve una secuencia clara.

Tabla 1. Propuesta de descriptores para cada nivel de competencia de la orientación espacial

\begin{tabular}{|c|c|}
\hline Nivel de competencia & Descriptores \\
\hline $\begin{array}{l}\text { Ubicación espacial y } \\
\text { trayectoria intuitiva }\end{array}$ & $\begin{array}{l}\text { - Uso de claves internas sensoriomotrices, construidas con base en la } \\
\text { posición personal e intuiciones de distancia y dirección, para ubicar } \\
\text { objetos, personas o lugares. } \\
\text { - Construcción de evocaciones mentales, con base en claves internas } \\
\text { - } \text { - Consoriomotrices para retener ubicaciones y trayectorias. } \\
\text { ambiente cercano o a la vista, para ubicarse y reproducir trayectorias. } \\
\text { - Uso de claves externas, construidas con base en la posición personal e } \\
\text { intuiciones de distancia y dirección, para integrar ubicaciones a partir de } \\
\text { relaciones de cerradura, conectividad y continuidad. } \\
\text { - Representación gestual, principalmente con las manos para representar } \\
\text { ubicaciones y direcciones. }\end{array}$ \\
\hline Organización espacial & $\begin{array}{l}\text { - Construcción de claves externas, constituidas por objetos familiares o } \\
\text { no familiares, que están o no a la vista, para ubicar objetos, personas o } \\
\text { lugares, reproducir trayectorias y planear rutas eficientes. } \\
\text { - Uso de sistemas de referencia concretos, construidos mediante un } \\
\text { conjunto de claves externas independientes de la posición personal para } \\
\text { ubicar objetos personas o lugares y describir trayectorias. } \\
\text { - Construcción de imágenes mentales, con base en claves externas para } \\
\text { identificar y describir ubicaciones y trayectorias. } \\
\text { - Representación icónica de ubicaciones y trayectorias sin tener en cuenta } \\
\text { - escalas. } \\
\text { - } \text { qunificación de rutas eficientes en situaciones que implican ubicaciones } \\
\text { - Localización de ubicaciones y planificación de trayectorias mediante el uso } \\
\text { de mapas icónicos. }\end{array}$ \\
\hline Modelos y mapas & $\begin{array}{l}\text { - Uso de sistemas de referencia representacionales, a partir de modelos y } \\
\text { escalas para ubicar objetos o lugares y describir o planificar trayectorias } \\
\text { eficientes. } \\
\text { - Uso de correspondencia geométrica (congruencia y semejanza) entre } \\
\text { mapas o modelos a escala y el espacio real, para identificar ubicaciones } \\
\text { de objetos o lugares y describir trayectorias. } \\
\text { - Creación de representaciones a escala, para establecer relaciones de } \\
\text { distancia, de ubicaciones de objetos o lugares y trayectorias. (Primer nivel } \\
\text { de matematización). } \\
\text { - Uso de símbolos para representar el espacio real en modelos. } \\
\text { - Localización de ubicaciones y planificación de trayectorias mediante el uso } \\
\text { de mapas a escala. }\end{array}$ \\
\hline $\begin{array}{l}\text { Coordenadas y } \\
\text { estructuración espacial }\end{array}$ & $\begin{array}{l}\text { - Uso de sistemas de coordenadas euclídeas o polares para representar y } \\
\text { describir ubicaciones y trayectorias. } \\
\text { - Planificación de trayectorias eficientes en sistemas de coordenadas } \\
\text { euclídeas o polares, con base en relaciones de orden y distancia. } \\
\text { - Localización de ubicaciones y planificación de trayectorias en sistemas de } \\
\text { coordenadas euclídeas o polares. }\end{array}$ \\
\hline
\end{tabular}


Fuente: elaboración propia.

\section{Juegos didácticos asociados a los niveles de la competencia}

La propuesta didáctica consta de cuatro juegos diferentes inspirados en el juego Ricochet Robots ${ }^{4}$, en los que se busca planificar un tipo de trayecto eficiente, para desplazarse de un lugar a otro, según reglas para el movimiento y cambio de dirección. A continuación, describimos sucintamente los juegos. En Poloche y Zapateiro (2016), se encuentra una explicación más detallada de las reglas de cada uno.

\section{Primer juego Caminando a Ciegas}

Este juego consta de una cuadrícula de $8 \times 8$ dibujada en el piso o sobre un tapete, nueve obstáculos que simbolizan barreras que impiden el paso, y un banderín que representa la meta (figura 1).

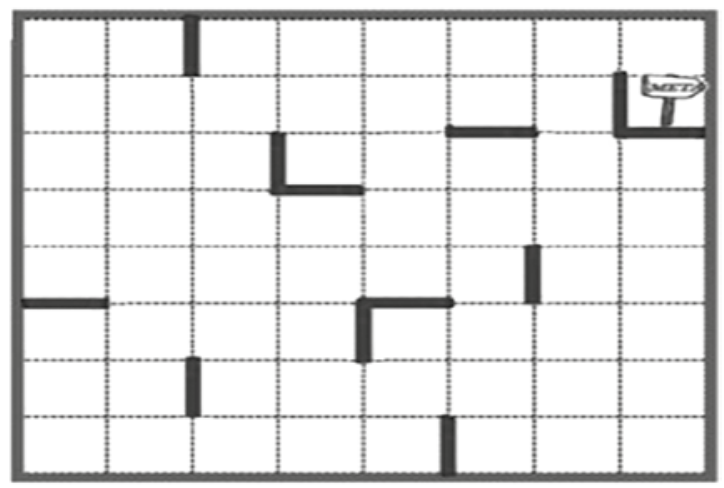

Figura 1. Cuadrícula juego uno

El juego comienza con la observación de un trayecto, planeado y ejecutado por el director del juego, es decir el docente, a partir de la posición personal (figura 2). Posteriormente,

4 Ricochet Robots es un juego de mesa, diseñado por Alex Randolph. Este juego fue publicado por primera vez en Alemania como Rasende Roboter, en el año 1999 (tomado de Wikipedia, 2016, https://en.wikipedia.org/ wiki/Ricochet_Robot

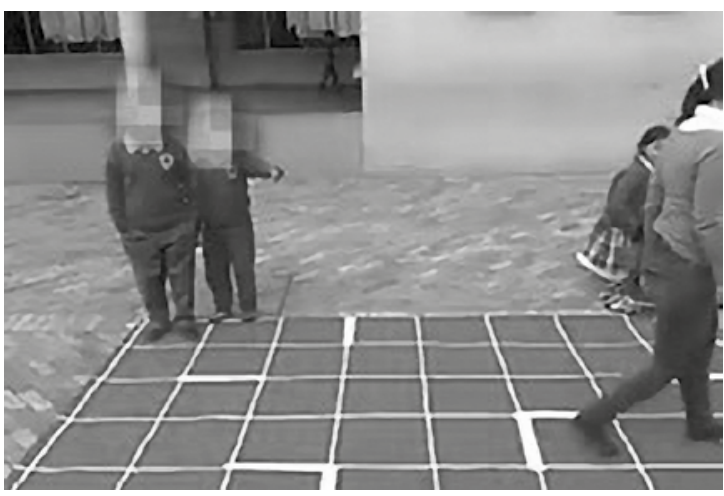

Figura 2. Observación trayecto

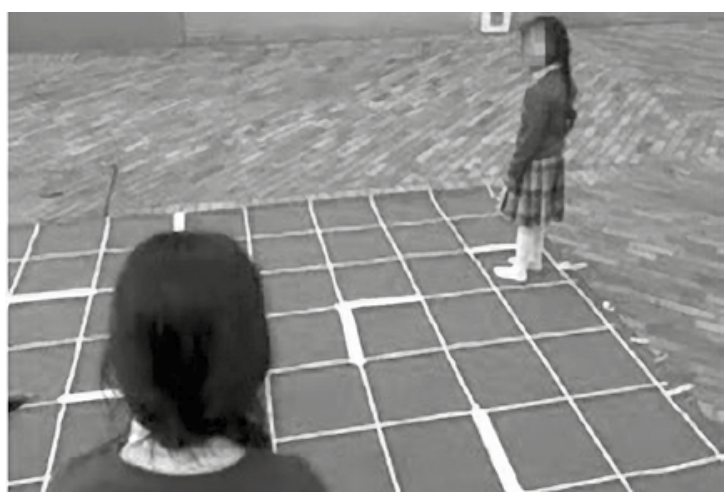

Figura 3. Trayecto-ojos abiertos

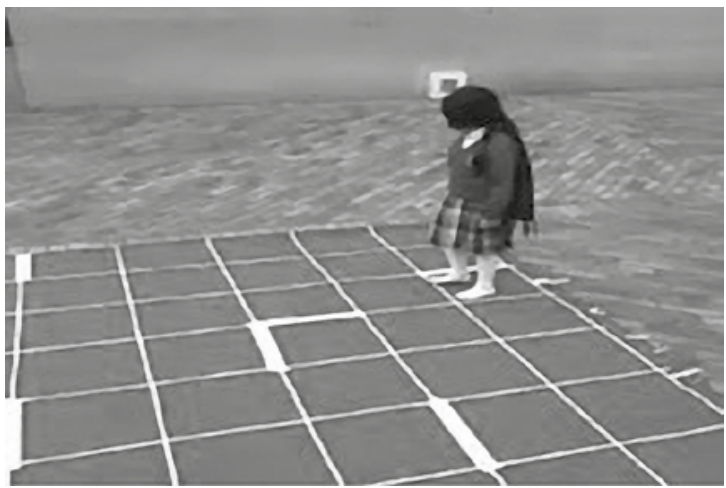

Figura 4. Trayecto-ojos vendados

Las trayectorias se realizan caminando en línea recta, sin avanzar por diagonales, hasta encontrar algún obstáculo, o hasta llegar a un borde externo de la cuadrícula. Cuando se llega a un obstáculo o a un borde externo, se debe detener el movimiento y girar $90^{\circ}$ para tomar una nueva dirección. Este proceso se 
repite tantas veces se requiera para llegar a la meta. (ver ejemplos de trayectos en la figura 5). Durante el recorrido, se requiere describir en voz alta los movimientos y direcciones que se van tomando. Por ejemplo, "giro a la derecha", "avanzo de frente hasta el obstáculo y giro hacia la izquierda". Gana un punto quien reproduzca la trayectoria correctamente con los ojos abiertos y con los ojos vendados.
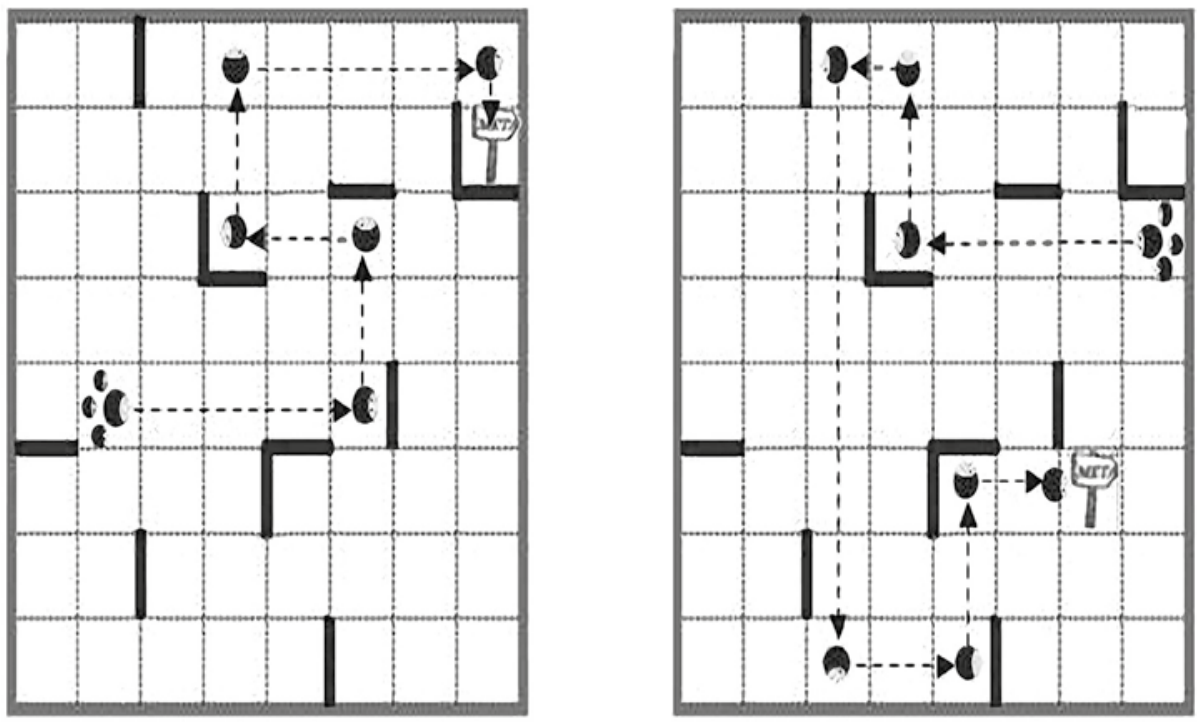

Figura 5. Ejemplos de trayectorias del juego Caminando a Ciegas

Durante el juego es importante que el docente promueva la identificación de relaciones de dirección y distancia, para que los estudiantes puedan orientarse y describir movimientos, desde el punto de vista de su posición personal. Caminando a Ciegas contribuye al desarrollo del nivel de ubicación espacial y trayectoria intuitiva, ya que se requiere identificar la posición personal en el espacio de juego, con respecto a la ubicación del banderín y los obstáculos. A través de la observación, los estudiantes pueden retener la ruta y construir claves externas, con respecto a un punto de referencia (el jugador mismo), la distancia estimada a los objetos (lejanía o cercanía) y la dirección (izquierda, derecha, adelante o atrás). Cuando los estudiantes reproducen con los ojos abiertos la trayectoria observada, construyen evocaciones mentales con base en la propia posición y sus movimientos (claves internas), registrando distancias aproximadas y direcciones, que pueden ser útiles a la hora de reproducir el trayecto con los ojos vendados. 
Segundo juego Destino Saturno

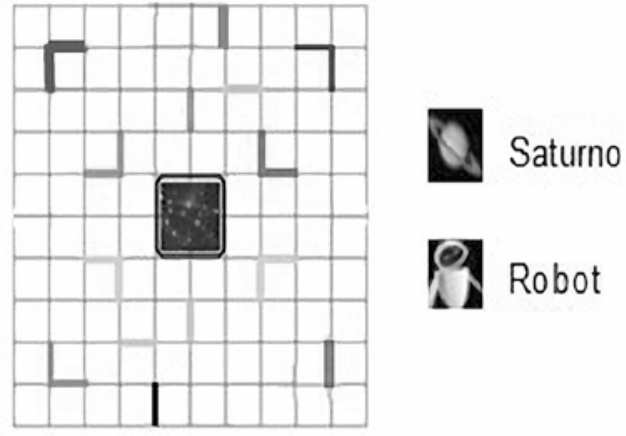

Figura 6. Tablero juego dos

Este juego consiste en planificar trayectos eficientes para llegar a una meta, a partir del punto de vista de otro observador (un robot). Las trayectorias se recorren de la misma forma que el juego Caminando a Ciegas (figura 7).
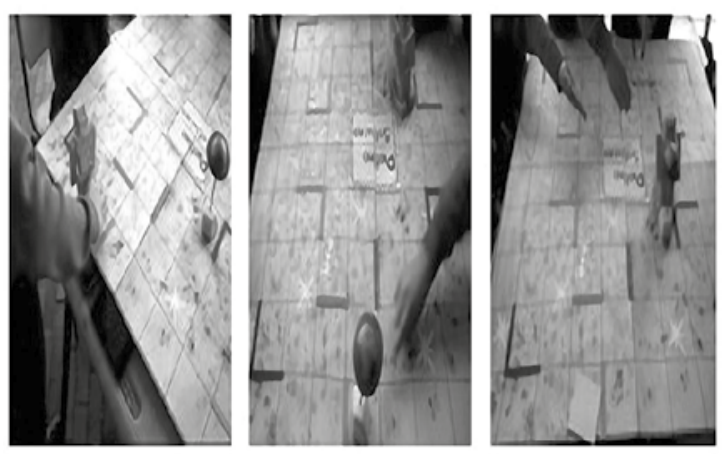

Figura 7. Ejecución de trayectos eficientes

Para describir los movimientos del robot, se debe tener en cuenta la posición de este y no la ubicación personal. Los jugadores compiten proponiendo un trayecto con el menor número de movimientos para que el robot llegue a Saturno, con el fin de promover la identificación de trayectorias eficientes. El total de recorridos hechos, contados entre cambio y cambio de dirección, determina el número de movimientos (ver ejemplos en figura 8). Gana un punto quien planee, ejecute y describa la trayectoria más corta.

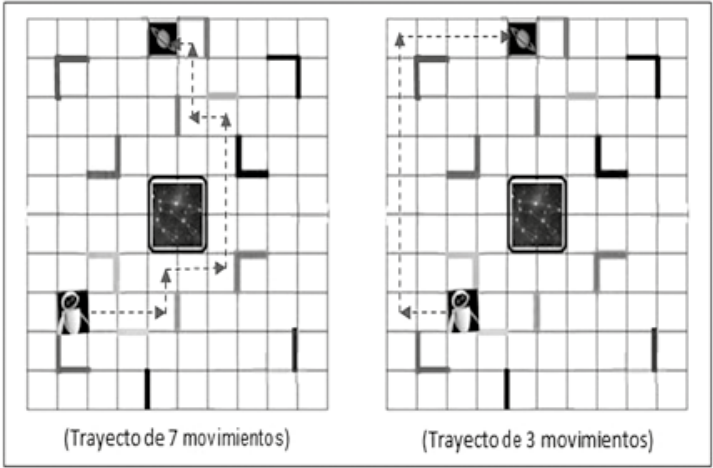

Figura 8. Ejemplos de trayectorias del juego Destino Saturno

Durante el juego se sugiere que el docente plantee cuestionamientos acerca de la perspectiva que cada jugador tiene de la ubicación del robot en el tablero, y promueva la descripción verbal de los trayectos, con el fin de favorecer la adquisición de competencias comunicativas para indicar ubicaciones o rutas. Destino Saturno contribuye al desarrollo del nivel de organización espacial, ya que requiere construir claves externas independientes de la posición personal. Cuando los estudiantes planean mentalmente la trayectoria más eficiente, construyen imágenes mentales de todos los posibles caminos o trayectos para llegar a la meta. Esta construcción implica conectar puntos de referencia externos para poder comparar, elegir y ejecutar el trayecto efectivamente.

\section{Tercer juego Perdido en Chiarezza}

El juego consta de una cuadrícula de $12 \times 12$, una serie de obstáculos e imágenes representados en un tablero (figura 9), cuatro robots de diferentes colores, hojas con un bosquejo del tablero (figura 10) y cuarenta fichas objetivo que se extraen al azar (figura 11). Cada una de estas fichas tiene dibujada en su superficie una de las imágenes del tablero, que representa la meta; el color de la ficha indica el robot que está en juego. 


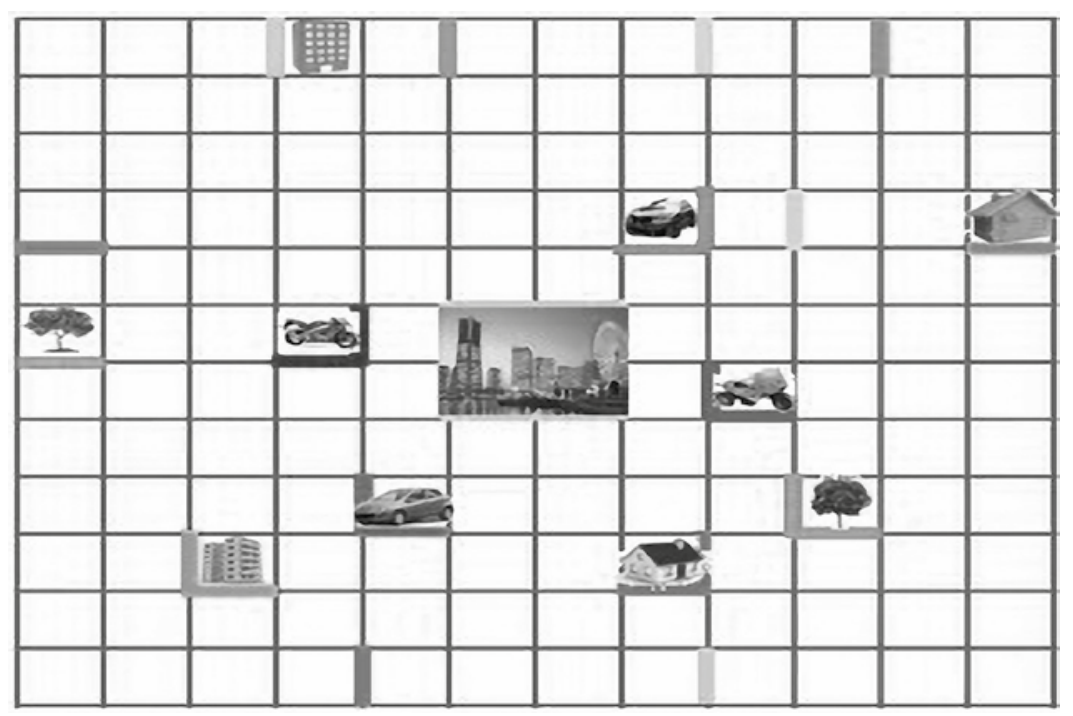

Figura 9. Tablero juego tres

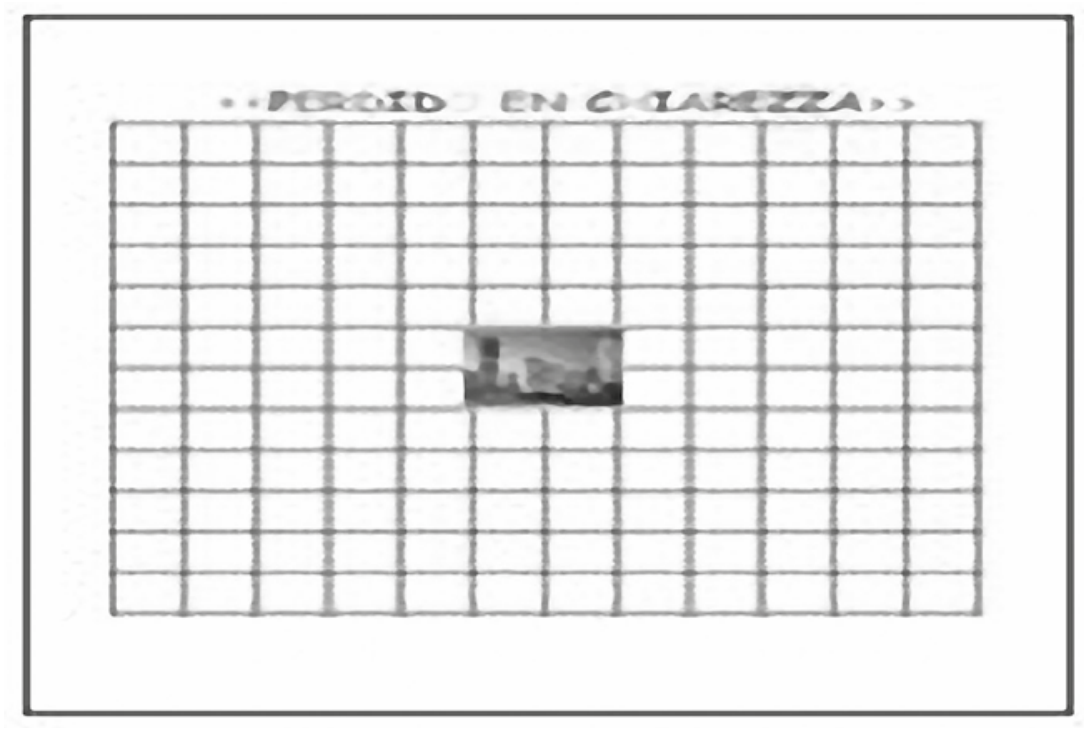

Figura 10. Bosquejo tablero 


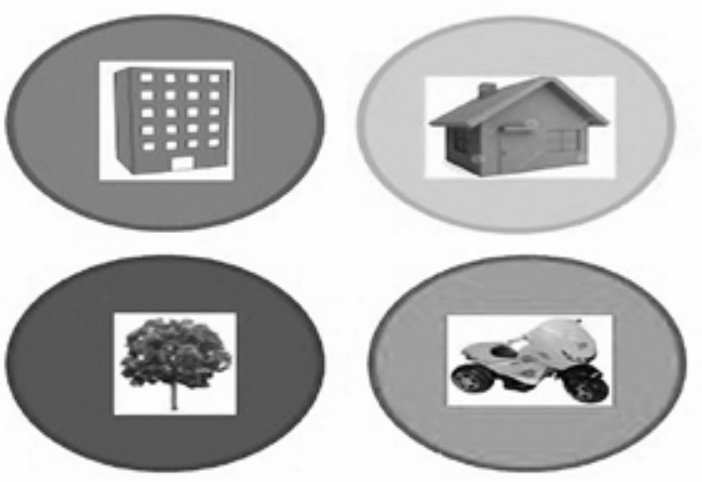

Figura 11. Ejemplo fichas objetivo

El juego comienza con la elaboración de un mapa del trayecto del robot, para ir del lugar en donde está inicialmente, al lugar señalado por la ficha que define la meta de la partida. Un compañero de juego, quien no tiene conocimiento previo del trayecto planeado, ejecuta el movimiento representado (figura 12).
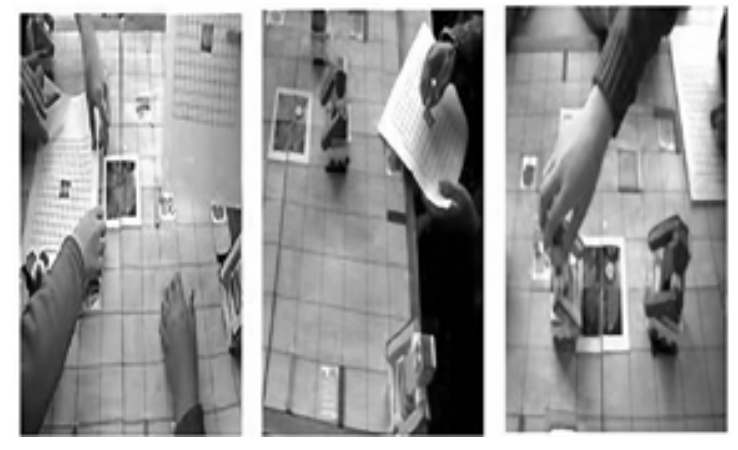

Figura 12. Elaboración y ejecución de trayectos a través de mapas

El orden de participación para ejecutar la trayectoria usando el mapa se establece de acuerdo con el jugador que tenga el menor número de movimientos en la ruta (ver ejemplo en figura 13). Gana un punto la pareja que dibuje sobre la hoja el mapa del trayecto más eficiente y usando el robot ejecute y describa la trayectoria indicada en el mapa.

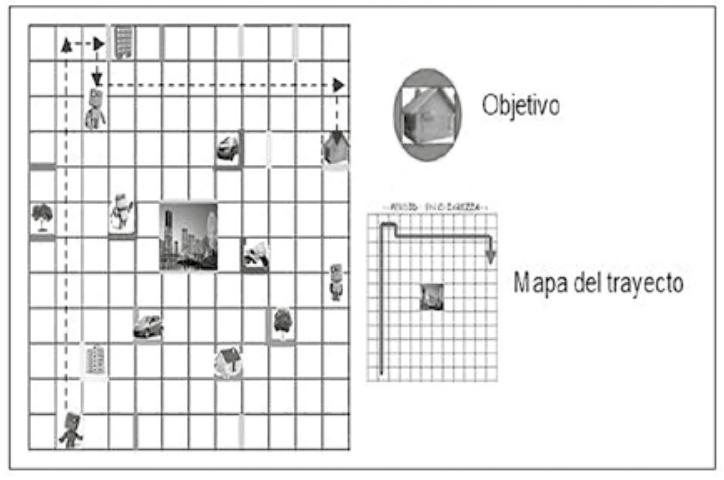

Figura 13. Ejemplo de trayectoria del juego Perdido en Chiarezza

Para que el juego tenga un impacto positivo en la enseñanza y aprendizaje de la orientación espacial, es necesario que el docente incentive el análisis de la ubicación de los objetos del tablero de juego en relación con los mapas, para que los estudiantes puedan usar relaciones de correspondencia. Perdido en Chiarezza contribuye al desarrollo del nivel de modelos y mapas, ya que se requiere identificar la posición y ubicación de cada uno de los objetos representados y ubicados en el tablero de juego y seleccionar claves externas para crear relaciones de distancia, de ubicaciones de lugares y objetos; con estos se construye una representación a escala de una ruta, y se ejecutan trayectos a través de un mapa, por medio de la correspondencia geométrica y el uso de sistemas de referencia representacionales.

\section{Cuarto juego Rescatando Animales}

El juego consta de un tablero con una cuadrícula de $14 \times 14$, un conjunto de obstáculos simbolizados por espirales, algunas señales en forma de $x$ que indican que allí no pueden ubicarse objetos (figura 14), cuatro fichas de juego (rescatadores de animales), 24 piezas de animales, 24 tarjetas objetivo que indican el 
animal a rescatar (figura 15), y 124 tarjetas de ubicación que permiten identificar en dónde situar la ficha de juego (figura 16). Tanto las tarjetas objetivo como las de ubicación se extraen al azar.

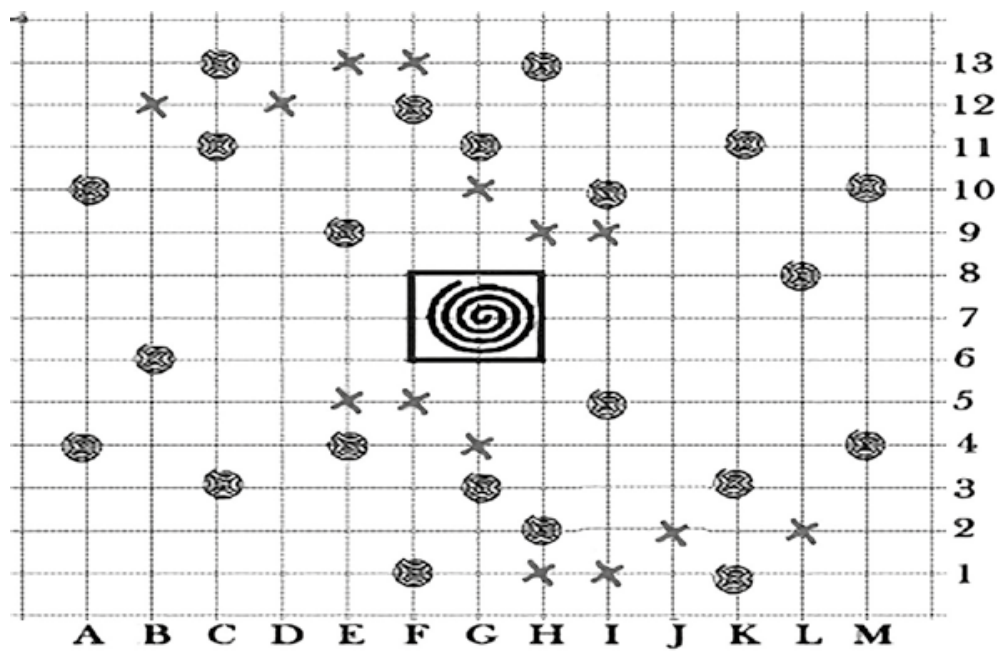

Figura 14. Tablero juego cuatro

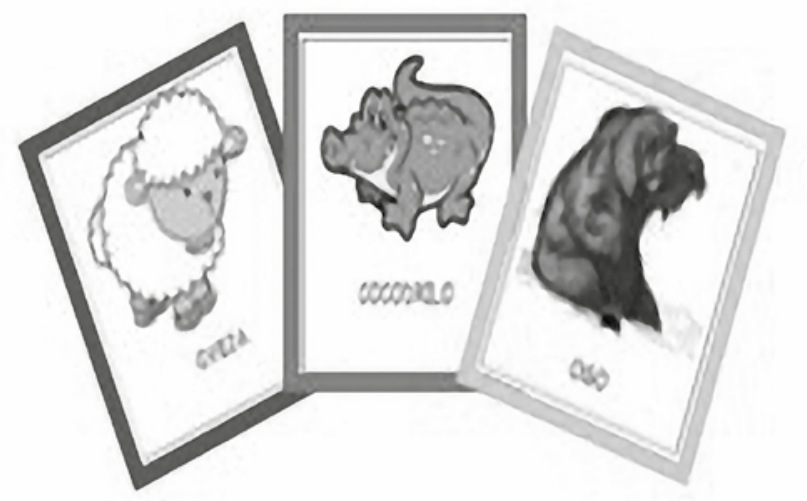

Figura 15. Tarjetas objetivo

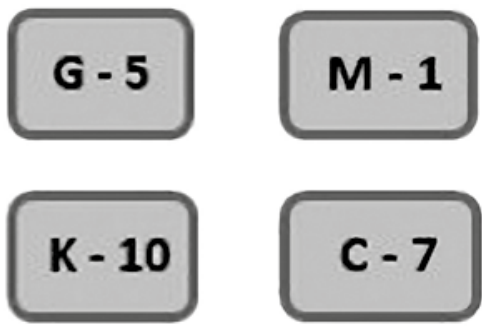

Figura 16. Tarjetas de ubicación 
El juego consiste en la identificación de ubicaciones para localizar objetos, planificar y ejecutar trayectorias eficientes en un sistema de coordenadas cartesianas (figura 17).
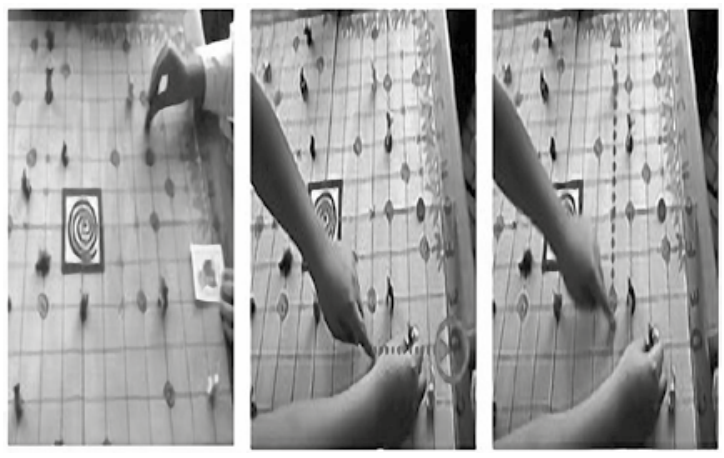

Figura 17. Identificación de ubicaciones en un sistema de coordenadas

Las fichas de juego se mueven sobre las líneas de la cuadrícula, con el fin de usar coordenadas cartesianas para identificar y describir ubicaciones en la ejecución de un trayecto (ver ejemplos en figura 18). Gana el jugador que logre ejecutar y describir correctamente el mayor número de trayectos eficientes para recuperar animales.
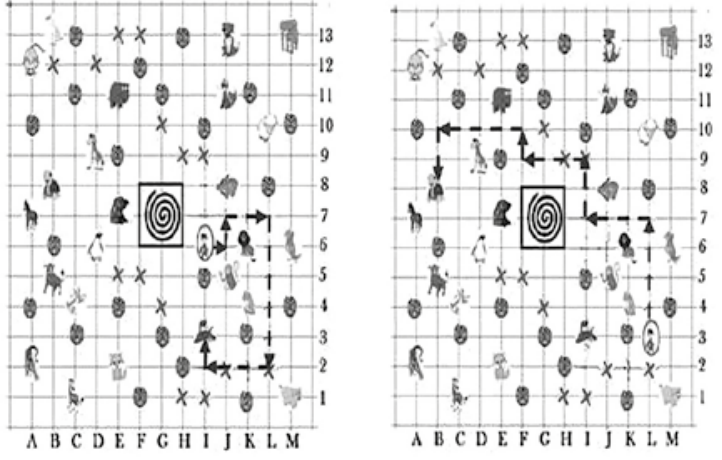

Figura 18. Ejemplos de trayectorias del juego Rescatando Animales

Se sugiere que, durante el desarrollo del juego, el docente promueva la identificación de las coordenadas en las que se ubican los animales y los "rescatadores", para que los estudiantes puedan aprender a especificar la ubicación de un objeto o lugar y describir la trayectoria que se debe recorrer para alcanzar el objetivo. Además, conviene que el docente plantee cuestionamientos acerca de lo que representan las etiquetas (letra, número) del tablero, para que los estudiantes puedan empezar a reconocer las coordenadas como una forma de organizar el espacio. El juego Rescatando Animales contribuye al desarrollo del nivel de coordenadas y estructuración espacial, ya que requiere que los estudiantes reconozcan los puntos de la cuadrícula del tablero de juego, por un par de etiquetas ordenadas mediante dos rectas numéricas perpendiculares, para que puedan localizar las ubicaciones que codifican una ruta, representar y describir un trayecto, teniendo en cuenta relaciones de orden en términos de izquierda, derecha, subir y bajar en el plano.

\section{Reflexiones sobre la implementación de los juegos en una experiencia piloto}

Cada uno de los juegos fue implementado a manera de prueba piloto, con estudiantes de grados tercero y quinto de primaria, de la Escuela Normal Superior Distrital María Montessori, sede A, ubicada en la localidad 15-Antonio Nariño de Bogotá, Colombia. Una autora del artículo dirigió los juegos, mientras la otra hizo una grabación en video. Con ello, buscamos identificar el impacto que tenían los juegos en los niños con relación a la competencia orientación espacial.

A partir de un ejercicio analítico acerca de lo sucedido durante las pruebas piloto, detectamos aspectos positivos respecto al desarrollo de la orientación espacial, y a los juegos como recurso didáctico. También evidenciamos algunas dificultades a tener en cuenta por quienes estén interesados en 
usarlos. En la tabla 2 relacionamos algunos de los hallazgos obtenidos de cada uno de los juegos de la propuesta, en correspondencia con los descriptores que caracterizan cada uno de los niveles de competencia de la orientación espacial.

Tabla 2. Aspectos de la orientación espacial que se desarrollaron

a través de los juegos

\begin{tabular}{|c|c|}
\hline Juego & Aspectos de la orientación espacial \\
\hline $\begin{array}{l}\text { Caminando } \\
\text { a Ciegas }\end{array}$ & $\begin{array}{l}\text { - Desarrollo de habilidades simbólicas. } \\
\text { - Construcción y uso de claves externas con base en la posición personal. } \\
\text { - Uso de relaciones topológicas. } \\
\text { - Codificación de trayectos. } \\
\text { - Uso de la memoria perceptiva. }\end{array}$ \\
\hline $\begin{array}{l}\text { Destino } \\
\text { Saturno }\end{array}$ & $\begin{array}{l}\text { - Localización de objetos. } \\
\text { - Planeación y ejecución de trayectos eficientes. } \\
\text { - Construcción y uso de claves externas independientes de la posición } \\
\text { personal. } \\
\text { - Descripción de ubicaciones y trayectos desde el punto de vista de otro } \\
\text { observador. }\end{array}$ \\
\hline Perdido en Chiarezza & $\begin{array}{l}\text { - Uso de la correspondencia geométrica para localizar objetos y hacer } \\
\text { recorridos. } \\
\text { - Planeación y ejecución de trayectos eficientes. } \\
\text { - Creación de representaciones a menor escala. } \\
\text { - Descripción de trayectos y ubicaciones a partir de un modelo o mapa a } \\
\text { escala. }\end{array}$ \\
\hline Rescatando Animales & $\begin{array}{l}\text { - Identificación de pares ordenados. } \\
\text { - Localización de ubicaciones en un sistema cartesiano de coordenadas. } \\
\text { - Descripción de trayectos mediante un sistema cartesiano de coordenadas. }\end{array}$ \\
\hline
\end{tabular}

Fuente: elaboración propia.

A continuación, presentamos un ejemplo del trabajo realizado. Para ello, transcribimos el diálogo sostenido con un niño al finalizar el juego Caminando a Ciegas.

Directora: ¿Cómo hicieron para moverse con los ojos cerrados?

Estudiante: Contando los pasos para ganar.

Directora: iContando los pasos para ganar! Y żcómo sabías que tenías que girar a la izquierda o a la derecha?

Estudiante: Mecánicamente.

En la conversación evidenciamos que el estudiante logró hacer una evocación mental basada en claves internas sensoriomotrices. Cuando expresa que contó los pasos, vemos que codificó el trayecto, con base en el propio cuerpo y aproximaciones de distancia entre los puntos de referencia; es decir, entre los obstáculos de la cuadrícula. Por otra parte, cuando el niño comunica que se movió "mecánicamente", interpretamos que usó la memoria perceptiva, a partir de la experiencia de su propio movimiento cuando realizó el trayecto con los ojos abiertos.

Nos parece importante mencionar que durante el desarrollo del juego Perdido en Chiarezza, los estudiantes lograron hacer uso de la correspondencia geométrica 
entre la imagen del tablero de juego y la imagen hecha a menor escala, para dibujar y orientar exitosamente el trayecto. Contaron las casillas del tablero para identificar una ubicación en el mapa a menor escala, generando mecanismos propuestos por ellos mismos, para poder plasmar el espacio real en un modelo. Además, a través de las fichas objetivo, se fomentó la utilización de símbolos para desarrollar relaciones de correspondencia geométrica. Los niños identificaron mediante la imagen presentada en la ficha objetivo, atributos geométricos, como la forma del objeto y atributos físicos, como el color del objeto, para relacionar la imagen con uno de los objetos representados en el tablero. Este proceso puede ilustrarse con algunas expresiones verbales de los niños: "Es un carro" (correspondencia geométrica, mediante atributos geométricos de forma), "la moto es azul" (uso de atributos físicos para corresponder particularidades de un objeto). Con ello, se favoreció la comprensión de la correspondencia geométrica entre la información que brinda un modelo o un mapa, y la ubicación o la trayectoria de un objeto en el espacio real.

La principal dificultad detectada en la implementación de los juegos era que, en algunas ocasiones, los estudiantes no usaban la estructuración espacial del tablero de juego y se basaban en su posición espacial a la hora de comunicar los movimientos relacionados con una trayectoria. Al no emplear los puntos de referencia del tablero como una forma de organizar el espacio, daban información poco clara a sus compañeros. Por ejemplo, se referían a movimientos a la derecha, la izquierda, arriba o abajo, sin tener en cuenta el punto de vista de quien hacía el movimiento. Con orientaciones del director del juego y recomendaciones sobre la forma de comunicar información, la dificultad se fue sorteando poco a poco.

Teniendo en cuenta los resultados obtenidos, evidenciamos que los juegos diseñados aportan al desarrollo de la orientación espacial. En cada uno de ellos, se destacan habilidades referentes a los descriptores para cada nivel de la competencia. Por otra parte, identificamos que los cuatro juegos de la propuesta, despiertan el interés y la motivación de los estudiantes, además les aportan al desarrollo integral, emocional y social.

\section{Referencias}

Chamoso, J., Durán, J., García, J., Lalanda, J., y Rodríguez, M. (2004). Análisis y experimentación de juegos como instrumentos para enseñar matemáticas. Suma: Revista sobre la enseñanza y el aprendizaje de las matemáticas, (47), 47-58. Recuperado de http:// revistasuma.es/IMG/pdf/47/047-058.pdf

Gonzato, M., y Godino, J. (2010). Aspectos históricos, sociales y educativos de la orientación espacial. Revista Iberoamericana de Educación Matemática, 23, 45-58.

Gonzato, M., Fernández, T., y Díaz, J. (2011). Tareas para el desarrollo de habilidades de visualización y orientación espacial. Números: Revista de didáctica de las matemáticas, 77, 99-117. [Recuperado de la base de datos Dialnet].

Macías, G., y Quintero, R. (2013). Representaciones de recorridos en entornos virtuales de videojuegos comerciales. Actas del VII CIBEM, 1703-1710.

Ministerio de Educación Nacional (MEN) (1998). Lineamientos curriculares en Matemáticas. Bogotá: Gaia.

Ministerio de Educación Nacional (MEN) (2006). Estándares básicos de competencias en Matemáticas. Bogotá: Autor.

Ministerio de Educación Nacional (MEN) (2015). Derechos básicos de aprendizaje. Matemáticas. Bogotá: Autor. 
Muñiz, L., Alonso, P., y Rodríguez, L. (2014). El uso de juegos como recurso didáctico para la enseñanza y aprendizaje de las matemáticas: estudio de una experiencia innovadora. Unión: Revista Iberoamericana de Educación Matemática, 39, 19-33. Recuperado de http://www.fisem.org/www/union/revistas/2014/39/archivo6.pdf

Nerea, E. (2013). El juego y la Matemática. Juegos de matemáticas para el alumnado del primer ciclo de E. primaria (trabajo de grado). Universidad de Valladolid. España. Recuperado de http://uvadoc.uva.es/handle/10324/4809

Organización para la Cooperación y el Desarrollo Económicos (OCDE) (2013). Marcos y pruebas de evaluación de PISA 2012. Recuperado de http:// www.mecd.gob.es/dctm/inee/internacional/pisa2012/marcopisa20 12. pdf? documentld=0901 e72b8177328d

Organización para la Cooperación y el Desarrollo Económicos (OCDE) (2014). Resultados de PISA 2012 en foco: lo que los alumnos saben a los 15 años de edad y lo que pueden hacer con lo que saben. Recuperado de http://www.oecd.org/ pisa/keyfindings/PISA2012_Overview_ESP-FINAL.pdf

Poloche, S., y Zapateiro, J. (2016). Orientación espacial: una ruta de enseñanza y aprendizaje centrada en ubicaciones y trayectorias (trabajo de grado). Universidad Pedagógica Nacional, Bogotá.

Rodríguez, A. (201 1). Geografía conceptual: enseñanza y aprendizaje de la geografía en la educación básica secundaria. Bogotá: Grupo Geopaidea.

Sarama, J., y Clements, D. (2009). Early Childhood Mathematics Education Research: Learning Trajectories for Young Children. New York: Routledge.

Secretaría de Educación Distrital (SED) (2014). Análisis descriptivo y pedagógico de los resultados de las pruebas Saber 3..$^{\circ} 5^{\circ}$ y 9..$^{\circ}(2009,2012$ y 2013) y Saber 11 (2014). Bogotá: Subdirección de Calidad y Pertinencia Dirección de Evaluación de la Educación Secretaría de Educación del Distrito. Recuperado de: http:// repositoriosed.educacionbogota.edu.co/ispui/bitstream/123456789/6612/1/ Analisis_descriptivo_\%20pedagogigo_SABER_359_11.pdf

Tartre, L. (1990). Spatial Orientation Skill and Mathematical Problem Solving. Journal for Research in Mathematics Education, 21 (3), 216-229.

\section{Para citar este artículo}

Zapateiro, J., Poloche, S., y Camargo, L. (2018). Orientación espacial: una ruta de enseñanza y aprendizaje centrada en ubicaciones y trayectorias. Tecné, Episteme y Didaxis: TED, 43, 119-136. 